\title{
AN EFFICIENT RELAY SELECTION METHOD BASED ON ANN CHANNEL ESTIMATION TECHNIQUE FOR AMPLIFY AND FORWARD RELAY IN COOPERATIVE NETWORKS
}

\author{
EZHILAZHAGAN C ${ }^{1}$ And RAMAKRISHNAN M ${ }^{2}$ \\ ${ }^{1}$ Assistant Professor, Department of ECE, Dr.NGP Institute of Technology, Kalapatti Road, Coimbatore, \\ ezhisang20@gmail.com \\ ${ }^{2}$ Professor \& Dean, Department of ECE, Sethu Institute of Technology, Kariapatti, Virudhunagar, India. \\ ramtechus@gmail.com
}

\begin{abstract}
Owing to high degree of flexibility, Artificial Neural Networks (ANN) can be used to model nonlinear channel estimation phenomenon. An ANN-based channel estimation technique and relay selection scheme was proposed as an alternate to Fuzzy Logic Controller (FLC) based channel estimation system. ANN's learning property is fully exploited to decipher the deteriorated symbols through extreme faded networks. Similar to FLC channel estimation techniques, this technique is found to be more effective in increasing bandwidth. Simulated results for Multi-input Multi-output (MIMO) of $2 \times 2$ and $4 \times 4$ using Additive White Gaussian Noise (AWGN) and Rayleigh channel in terms of quantity (Mbps) compared with Signal to Noise Ratio (SNR) over a choice of 0 to $30 \mathrm{~dB}$. This illustrates the effectiveness of the knowledge proficiency of ANNs is $92.19 \%$ of AWGN and $94.62 \%$ of Rayleigh whereas for FLC is $70.34 \%$ of AWGN and $75.51 \%$ of Rayleigh channel.
\end{abstract}

Keywords: Cooperative systems; Relay selection scheme; Amplify and forward; FLC; ANN; Channel estimation

\section{I.INTRODUCTION}

In view of Wireless Communication systems, multi-route fading is the important drawback to attain higher data rate. Frequency variation, time variation and spatial variation methods are determined to relieve the multi-route fading. Using spatial variation for communication yields more degree of freedom gain, which generally advances the scheme by presenting independent way communication. The model was attained by familiarizing several antennas and MIMO which advances the active manifolds. Because of cost, size and certain hardware restriction, real time system development is an added problem. Aimed to conquer these drawbacks, the cooperative communication system was presented in as a simulated MIMO state (Politis, C., et al., 2004).

For independent way communication which exists for practical operation, uses the transmission environment of the wireless communication where endpoint relays are presented between source and destination, which is an exact logic, are not in excess of forwarding the source signal built on certain strategic procedure (Laneman, J.N., et al., 2004). Using Space-Time Block Coding (STBC) with the advantage of MIMO spatial diversity settings appears to deliver better output on large fading wireless channels (Kharat, P. et al., 2013, Laneman, J.N., et al., 2003, and Hasna, M.O., et al., 2003). Nevertheless, Alamouti's space-time coding system depends on the accessibility of the right Channel State Information (CSI) source. If the channel estimation assistance is included in the method, the general presentation in setting up ties can be additionally improved. 
In MIMO systems, there are two common techniques that can be separated as blind and non-blind methods in the channel estimation literatures (Zhao, Y., et al., 2006). Blind estimation technique is computationally exhaustive than non-blind estimation, but the latter produces loss of reduced bandwidth by incorporating pilot symbols alongside real information sequence as training sequences. The use of ANNs for wireless communication has gained momentum in recent years, demonstrating its efficacy in solving many wireless transmission difficulties (Zhao, Y., et al., 2006). ANNs can be successfully applied to model nonlinear channel assessment phenomenon as it very well utilized to form randomly made nonlinear margin decision to take up complex characterizations. ANNs can carry out intelligent technical mapping of the input and output of the system; constructing various network models found effective response to find optimal channel estimation problem. Sowjanya Ponnaluru et al., (2020) estimated the channel in orthogonal frequency division multiplexing using deep learning applications. In Krikidis, I et al., (2008), the channel for MIMO systems was predicted using a three-layer ANN. Similar research work in different times has shown the significance of ANN in carrying out estimation tasks (Duong, T.Q., et al., 2009 and $\mathrm{Su}, \mathrm{W}$., et al 2005).

This paper recommends an effective channel assessment technique with feedback-based Artificial Neural Network (ANN) as an option in contrast with bandwidth-inefficient, pilot-based channel assessment procedure over Rayleigh and AWGN channels for MIMO systems. To decipher the deteriorated symbols over highly fading paths, the learning property of ANNs is completely misused. Simulated effects are observed in terms of SNR over a range of 0 to $30 \mathrm{~dB}$ versus quantity (Mbps) and the performance is analyzed for the proposed channel estimation technique based on ANN. This illustrates the utility of ANN's learning ability to enhance the channel estimation process through wireless fading networks.

\section{STRUCTURE MODEL}

Considering cooperative communication, the device model considered in this paper consists of a source $(\mathrm{S})$ that transmits its information to destination (D) that collaborates with $\mathrm{N}$ relays as shown in Figure 1(Ezhilazhagan,C et al., 2015). The standard model from source to destination uses direct transmission, i.e. whenever the receiver receives the signal it has to decode the information only based on the direct signal strength. If the straight transmission as of source to destination is not fully effective, then source- relay and the relay-destination i.e. data forwarded through the relay using a different path is considered. The cooperative network considers the other signal as a contribution in decoding the information by combining two signals. With the assumption that a single antenna will be connected to each terminal, it is believed that all relays working in half-duplex method considering $h_{s-o}, h_{s-r i}$, and $h_{r i-o}$ are the channel factors correspondingly from source to destination, source to $\mathrm{ri}^{\text {th }}$ relay, and from $\mathrm{ri}^{\text {th }}$ relay to destination. This one is similarly presumed that at each terminal there is ' 0 ' mean white Gaussian noise. As per the system model it is double hop, meaning that there is not any inter-relay contact and the source identifies all the relays channel information using the feedback loop. It is considered that the source transmission power is $E_{s}$ and $E_{r i}$ is the ith relay's transmission power (Sureshkumar K, et al., 2015). At 
this instant let ' $\mathrm{X}_{\mathrm{i}}$ ' be the information transmitted by the source and ' $\mathrm{Y}_{\mathrm{o}}$ ' be the received signal at the destination and denoted as:

$$
Y_{o}=h_{s-o} * \sqrt{E_{s}} X_{i}+n_{o}
$$

where $\mathrm{n}_{\mathrm{o}}$ is the noise at the destination.

At this time the received signal at the relay will be:

$$
Y_{r i}=h_{s-r i} * \sqrt{E_{s}} X_{i}+n_{r i}
$$

where $\mathrm{n}_{\mathrm{ri}}$ is the noise at the relay

Then, as per the relay protocol Amplify and Forward (AF), an intensified replica of the received signal is amplified by a relay element, will be transmitted to the destination that can be described as

$$
Y_{O-r i}=h_{r i-o} * \sqrt{E_{r i}} \beta\left(h_{s-r i} * \sqrt{E_{s}} X_{i}\right)+h_{r i-o} * \sqrt{E_{r i}} \beta n_{r i}+n_{O}
$$

where $\beta$ is the amplifier gain

In order to avoid the interference among the signals received at the destination, two different time slot is followed. During first time period, source communicates signal to destination in addition to the relay. However in the second time period, amplified replica of the transmitted signal is conveyed by means of relay. Many replicas of the source signal are stored by the destination and the Maximum Ratio Combiner (MRC) process is used collectively, whereas any time delay during the transmission is omitted. MRC is the method used for diversity combining where signals from different mediums are added, the channel gain ought to be relative to root mean square value of the signal level and contrarily relative to the mean square noise value in the channel and diverse equivalent constants i.e. weights utilized for every channel. MRC tries to restore the received side signal to its original form.

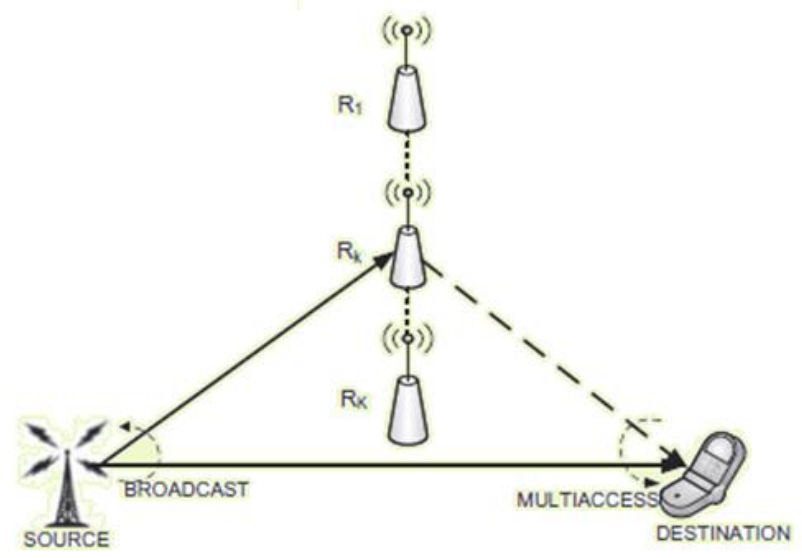

Figure 1. Cooperative Network Model

\section{a) Amplify and Forward Method}

The simple model of amplification and forward is shown in figure 1. AF is the co-operative signalling system's simplest principle (Krikidis, I., et al,. 2008 \& Ezhilazhagan,C., et al 2017) Every user gets added 
with noise of the signal from their significant other in this process; this noisy signal is then amplified and retransmitted.

\section{b) Relay choice built on Geographical statistics}

Wang C L., et al., (2009) were able to obtain relay selection based on geographical statistics with the goal of reducing the probability of symbol error. Geographical statistics determining the distance in the middle of source and relay is used as a measure to find the optimum relay for further communication. However, due to channel fading in addition to shadowing results that suggested procedure is not appropriate for realworld situation. Rong Wang et al., (2019) uses compressive channel estimation over the fast-fading channels. The three-time slot Time Division Multiple Access (TDMA) transmission procedure developed by the locality information was studied and investigated and improved performance was observed, compared to the old procedures. The procedural shortage is an additional time slot for broadcasting, which decreases the rate of code and is also impracticable due to locality-based information. Study of AF and Decode and Forward (DF) relaying activities for inter-relay contact with evidence in terms of SNR, has its own advantages which don't prove that AF can perform better than DF and vice versa.

\section{c) Relay choice built on Energy efficiency}

In the common situation, optimum energy transmission is recommended with the goal of improving the entire life cycle of the device. In addition, the optimum power distribution and the three principles of power-alert choice are evaluated. Relay choice with the impact of power-sharing procedure on the base of CSI for the purpose of providing an energy-saving relay choice approach is used (Madan R et al., 2008).

\section{d) Relay choice built on Outage probability}

Li Sun et al. will obtain the optimum-outage relay option method, by using the receiver's input on whether or not relay assistance is required. Adaptive DF and AF are also practiced as a transmission system (Desi Pramudiwati 2011). Nevertheless, equality based on outage value is predicted, with the intention of properly advancing relay option without showing trouble and enhancing it in network time (Nirmalkumar $\mathrm{S}$ et al., 2013). The outage performance of log-normal shadowing was examined to establish on outage likelihood using opportunistic DF (Mehlfuhrer C, et al., 2011).

\section{e) Relay choice built on Interference responsive}

Interference is an attractive communal which happens in a wireless system of communication and requires an inevitable result to end the act of communication in the instance of several transmission sets. Wenyuan Wang et al., (2019) investigated MIMO downlink channel estimation and the outcome of relay interference in cooperative systems. Wang C et al., (2009) obtained different transactions between disturbance control and cooperative relay scheme and dual spectrum selection methods. But the paper is 
not fully preparing the question of relay choice (Desi Pramudiwati 2011). Similarly, interference-based relay option is built to optimize joint knowledge on cooperative systems with a scarce destination for interference (Online). The choice of sensitive relay interference is suggested by Tanoli, U., et al (2012) using distributed interference-conscious relay choice technique in (Mehlfuhrer C, et al., 2011) to select the finest relay by means of intermediate node interference and channel information.

\section{f) Relay choice built on information from Channel state}

The main concerns not expressed in the paper were the network interval of option and not the exact protection of similar channel in data transmission and valuation length. Farooq Khan (2009) demand that the obsolete channel condition information be used to pick the finest relay and must be applied extremely a posteriori to measure the real SNR throughout the transmission and make sure operated it as a scheme for solitary relay choice. Instead of using the comprehensive information of the entire routes, Tanoli, U., et al (2013) used to relay the local channel conditions obtained at the relay and mark this one as the finest relay, thus executing dispersed relay option. SNR is intended with the obsolete SNR measurement technique, the size of the data transmission and the channel condition information obtained as the result of the recurrent relay choice process, where finest relay is selected, describing the relay selection procedure for wireless cooperative communication system based on fuzzy logic. This paper exploits fuzzy logic based algorithm on local CSI obtained by using the fuzzy logic method for computational complexity in order to reduce the repeated choice of relays. The FLC algorithm explicitly uses SNR, channel gain, and cooperative gain as three input variables for fuzzy logic.

The implementation of the proposed relay option structure using ANN is investigated for two fading networks, namely Rayleigh and AWGN, in connection with the twin phase cooperative method. In addition, a quantity of active relays and estimates of channels be determined by the selected threshold. Using Monte Carlo simulation, the presentations of both FLC centred relay selection and ANN constructed relay selection systems are calculated. Monte Carlo Simulation is used to design the probability of different outcomes in a process which cannot be easily predicted because of the presence of random variables. Hence this simulation can handle various range of problems virtually and also referred as multiple probability simulation.

\section{FLC Channel Estimation}

The minimum square channel estimator is attained by decreasing the square distance among the signal expected and the signal conveyed as follows.

$$
\min _{H^{T}} J(H)=\min _{H^{T}}\left\{|\overline{Y-\underline{X \bar{H}}}|^{2}\right\}
$$


$=\min _{H^{T}}\left\{(\bar{Y}-\underset{-}{X} \bar{H})^{T}(\bar{Y}-\underset{-}{X} \bar{H})\right\}$

where, $\overline{H^{T}}$ is the conjugate transpose operator.

We obtain equation (6) and (7) by distinguishing equation (5) with respect to $\overline{H^{T}}$ and calculating the minimum.

$$
\begin{aligned}
& \frac{\partial}{-} J(H)=-X_{-}^{T} \bar{Y}+X_{-}^{T} X_{-}^{T} X_{-} \bar{H}=0 \\
& \partial H^{T} \\
& \bar{H}=X_{-}^{-1} \bar{Y}=\left[\frac{Y_{O}}{X_{O}} \frac{Y_{1}}{X_{1}} \ldots \ldots . . . \frac{Y_{N C^{-1}}}{X_{N C^{-1}}}\right]^{T}
\end{aligned}
$$

FLC systems using channel estimation procedure is generally low in complication, but it is suffering due to high mean square error (MSE). A relay which outperforms another will be allocated for cooperative communication. The following factors are used to select the relay based on the algorithm:

\section{A. Signal to Noise Ratio}

The said is possible to write the straight path SNR, i.e. the propagation of a signal from source to equation (8).

$$
\gamma_{s-D}-r_{i}=\frac{\left|h_{s}-D\right|^{2}}{N_{D}}
$$

The SNR of the relay's first hop is represented by equation

$$
\gamma_{s}-r_{i}=\frac{\left|h_{s}-r_{i}\right|^{2}}{N_{r i}}
$$

In the second hop, the amplified signal is sent to a destination. The second relay hop SNR is defined as equation (10).

$$
\gamma_{r i-d}=\frac{\beta\left(h_{s-r i}\right)^{2}\left(h_{r i-D}\right)^{2}}{\left(\beta^{2}\left(h_{s-r i}\right)^{2}+1\right) N_{r i}}
$$

The required two hops SNR are applied for the relay option.

$$
\gamma_{i}=\min \left(\gamma_{s-r i,} \gamma_{r i-d}\right)
$$

\section{B. Co-operative Gain}


It is known as the proportion of bit error rate of the straight transmission $\left(\mathrm{BER}_{\text {direct }}\right)$ to bit error rate of the cooperative transmission $\left(\mathrm{BER}_{\text {cooperative }}\right)$. The relay BER used for the choice of relay can be expressed as equation (12):

$$
C G_{i}=\frac{\text { BERdirect }}{\text { BERcooperative }}
$$

\section{Channel Gain}

The least gain of the channel of two hops is deliberated for the proposed relay selection system. The gain of the channel used for relay choice selection is calculated using equation (13):

$$
h_{i}=\min \left(h_{s-r i}, h_{r i-d}\right)
$$

In figure 2 FLC consists of the relay selection system which has fuzzy input variables, fuzzification, Fuzzy Inference System (FIS), fuzzy rules, and defuzzification. The three input variables for fuzzy parameters are SNR, cooperative gain and channel gain. In the fuzzification method, variables are given a fixed value and the input membership functions are separated. The fuzzy input variables SNR takes five membership functions such as very small, small, intermediate, high and very high. The Cooperative Gain takes the value of three membership functions such as low, average and good. The channel gain takes the value of three membership functions such as worse, modest and best. So in evaluating the 5x3x3 fuzzy rules welldefined for it, the degree of significance are estimated with the strength of fuzzy rules and the output membership functions, as shown in figure. 3.

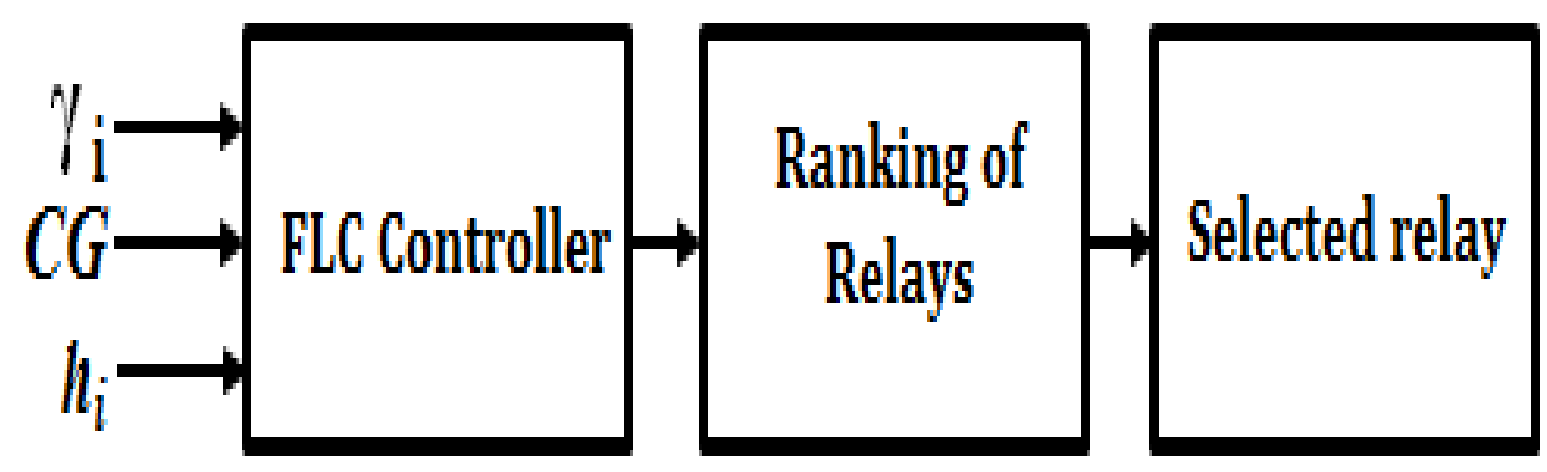

Figure 2. Function of FLC

The output membership function i.e. relay selection will have option such as (i) can be not selected, (ii) considered and (iii) selected. The Mamdani FIS is used in tandem with the fuzzification algorithms. The defuzzification uses the centroid method for estimating the output. 


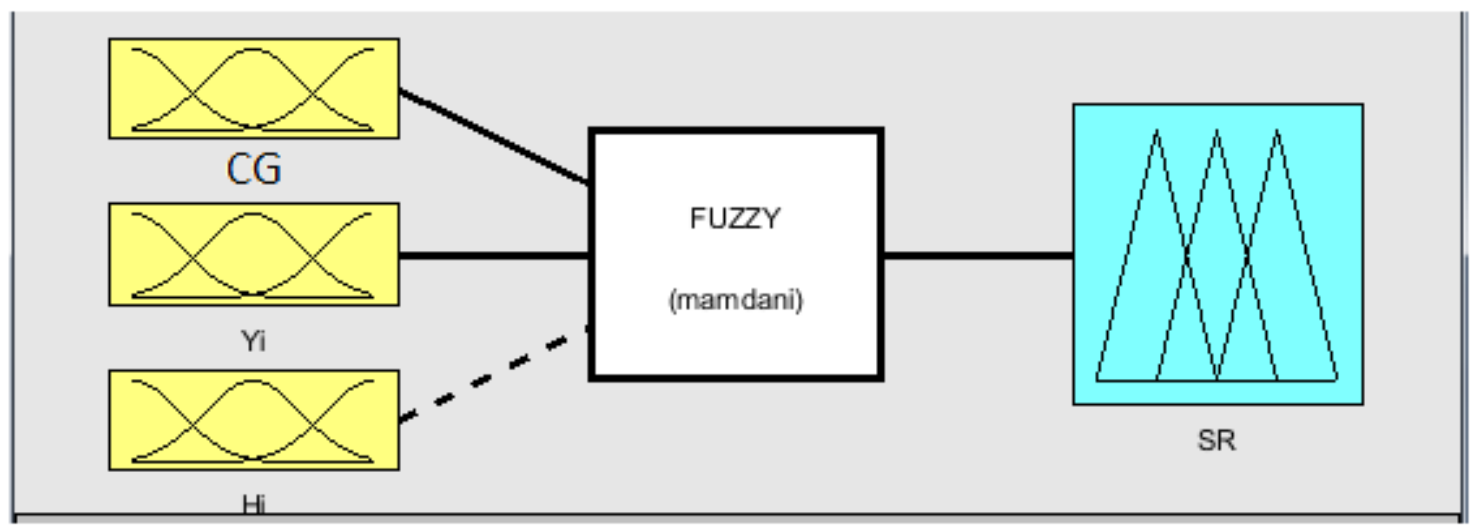

Figure 3. Fuzzification process of FLC

\section{Proposed ANN Based Channel Estimation}

ANN is the prototypical of the human brain that attempts to mimic the process of learning. ANN is a hugely equal appropriated processor comprising of essential preparing units, called neurons, which can learn and consequently sum up. An ANN accepts more amounts of inputs from the source or relays in the neural network. Each input comes with its weight and each neuron has its threshold value. Activating the neuron requires an activating signal called transfer function to generate the output of the neuron. The input neuron, hidden neuron and output neuron all three plays a role in the networks. These three layers are connected together to form a network. A simple network is a feed-forward network which doesn't have any closed path; the signal flow starting the input layer to hidden layer and reach the output layer called as Feed-Forward Neural Network (FFNN). Similar to FFNN, there is Back Propagation Neural Network (BPNN) where they have a weight connection with neurons in all three layers and fed back to the input layer.

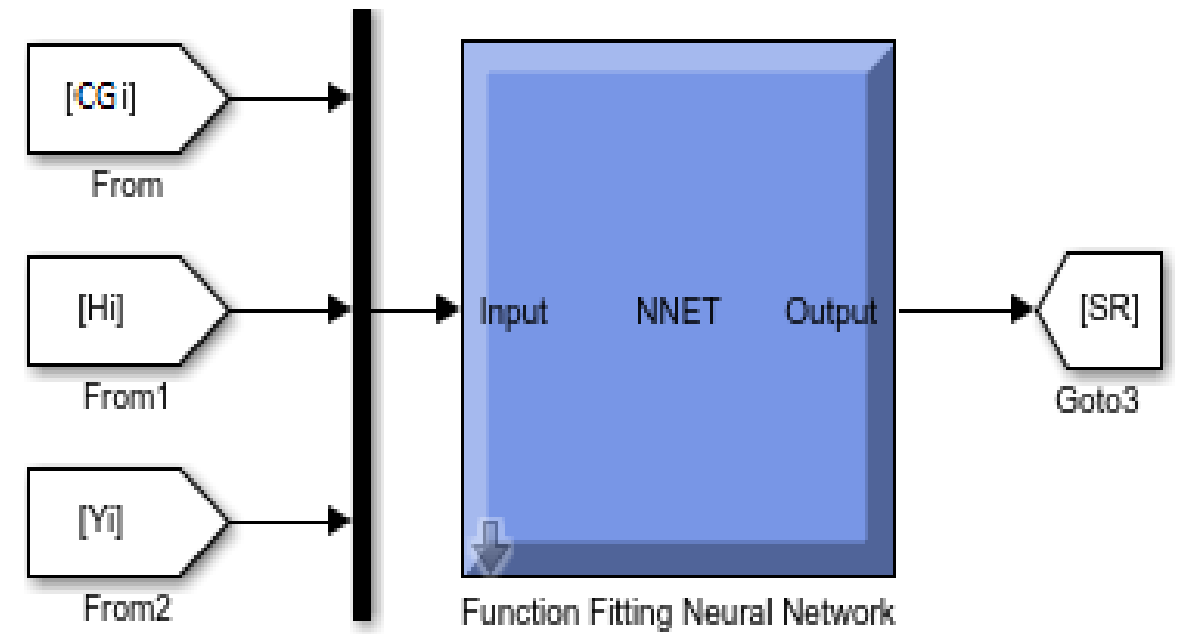

Figure 4. Trained ANN Structure 
A back propagation $\mathrm{ANN}$ is used in this research to find channel estimation and relay section using SNR, cooperative gain and channel gain. The $\mathrm{ANN}$ is used to evaluate in real time the optimal range of the relay, which corresponds to the input and output channel parameter. The set of data used to train the ANN was acquired after FLC. The functional neural network is shown in Figure 4 with an input layer of three inputs, a hidden layer of $n$ inputs and an output layer of one output. The established ANN configuration is a multilayer perceptron structure. In this collection of data, there are 1250 patterns of parameters such as SNR, Cooperative gain (CGi), channel gain (hi) and relay selection (SR) separated into two different sub databases, nearly 70 percent of the examples are used to train the ANN, and the remaining 30 percent are used to test and validate the system. A well-trained ANN will provide the reference voltage, very close to the desired value, as an output for a set of input parameters, resulting in a near-zero error of its $n$ inputs $\mathrm{v}_{\mathrm{k}}, \mathrm{k}=1,2,3 \ldots \mathrm{n}$ and producing an output as shown in Equation (14).

$$
a_{j}=\tan \operatorname{sig}\left(\sum_{k=1}^{n} w_{k} v_{k}+\text { bias }\right)
$$

The resulting weighted sum is given by the tan sigmoid function that regularly has a bias connected to it that can be viewed as an additional input.

The calculation of MSE value is specified in equation (15).

$$
e=\frac{1}{p} \sum_{i=1}^{p}\left\|y^{(i)}-v^{(i)}\right\|^{2}
$$

where,

$\mathrm{p}$ is number of information entries for training;

$\mathrm{y}$ is the vector output path for ANN;

$\mathrm{v}$ is the required output

\section{SIMULATION AND PARAMETERS}

The figure 5 shows simulated structure of cooperative network model, which is implemented for both FLC based channel estimation and ANN based channel estimation. Relation between the source and destination are deliberated in this scheme. The standard model looks at the source-to-destination connection, the relay source, and the relay destination. The coefficient of its channel is revealed by partnership and the AWGN is also exposed. Monte Carlo simulation is performed by considering relaying protocol as AF, Binary-Phase Shift Keying (B-PSK) as the modulation method for 2x2 and 4x4 MIMO scheme. 


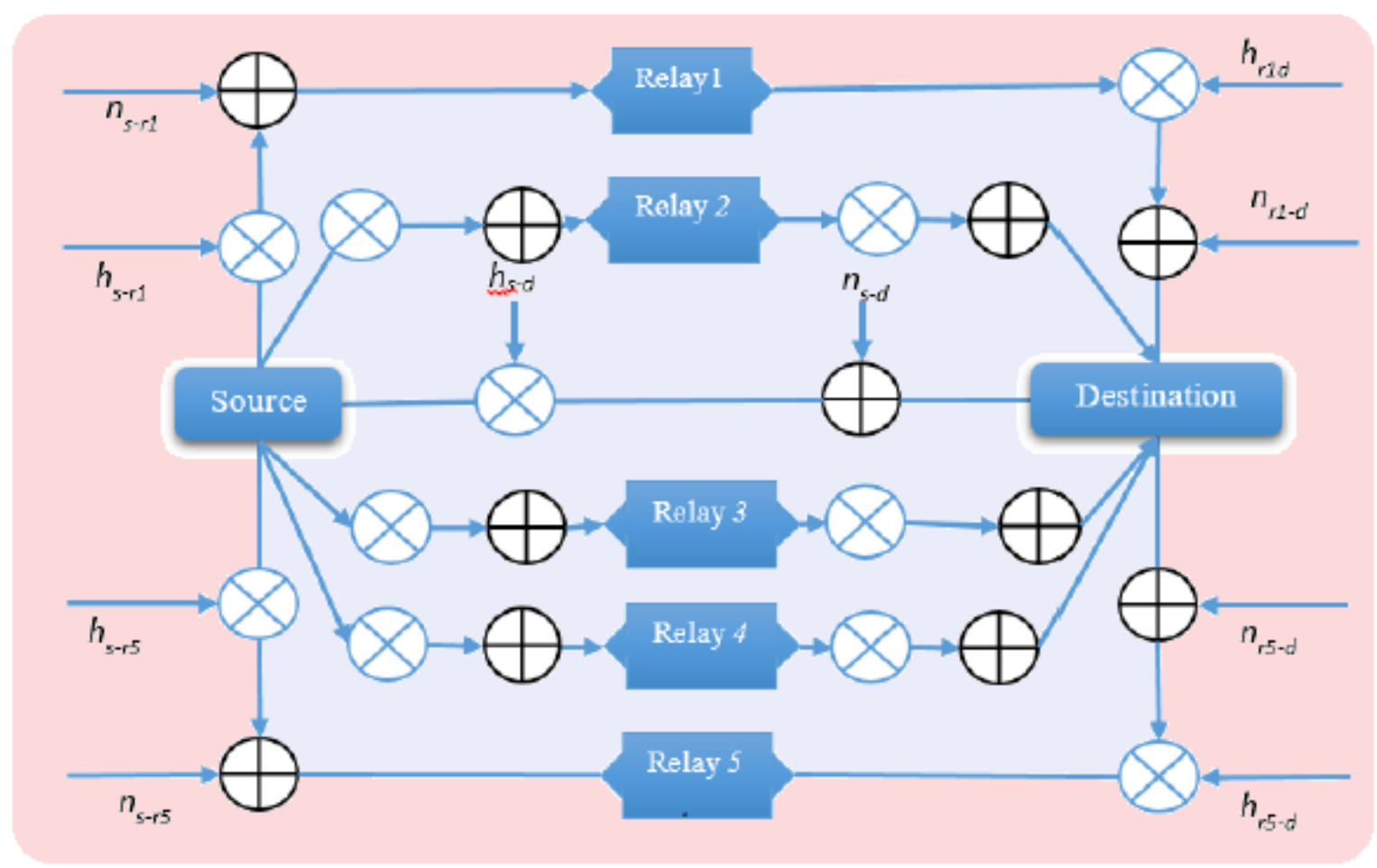

Figure 5. Simulated structure of Cooperative network model

Table 1. Simulation Bounds

\begin{tabular}{|l|l|}
\hline \multicolumn{1}{|c|}{ Parameters } & \multicolumn{1}{c|}{ Values } \\
\hline Transmitting Power of Source & 1 Watt \\
\hline Transmitting Power of Relay & 1 Watt \\
\hline Magnification Factor & Fixed \\
\hline Scheme of Modulation & B-PSK \\
\hline Antenna schemes & MIMO (2x2 and 4x4) \\
\hline Receiver Diversity & MRC \\
\hline Relays counts & 5 \\
\hline Relay Protocol & Amplify-and-Forward \\
\hline Bit value & $10^{\wedge} 6$ \\
\hline Receiver Noise & AWGN and Rayleigh \\
\hline
\end{tabular}

At each node, AWGN is applied and the full contact ratio is used as the receiver's diversity, transmitting bits of $10^{\wedge} 6$ for each transferred simulation from source to destination. All procedures are being studied for two types of networks, namely Rayleigh and AWGN. The simulation bounds and constraints for the proposed method are listed in Table I. 


\section{RESULTS AND DISCUSSION}

The target sample set is given to the ANN as the result of predictable path from the FLC complex type matrix. ANN's learning takes place during the training phase in which the ANN changes its weights according to the training algorithm. After that, ANN is fitted with 1250 generations in which the actual conventional FLC estimator measures the MSE between the estimated channel of ANN and the projected channel continuously. Ultimately, for the lowest MSE, channel estimation is calculated using stored weights in ANN, which provides the best ANN's estimated channel. The FLC produces approximate efficiency of $70.26 \%$ and ANN produces average efficiency of $94.63 \%$ compared with the ideal channel over the $2 \times 2$ AWGN channel.

Table 2. CL-MIMO - 2x2 using AWGN

\begin{tabular}{|c|c|c|c|c|c|c|c|}
\hline SNR (dB) & 0 & 5 & 10 & 15 & 20 & 25 & 30 \\
\hline $\begin{array}{c}\text { IDEAL } \\
\text { CHANNEL } \\
(\mathrm{Mbps})\end{array}$ & 1.021 & 2.491 & 4.198 & 6.848 & 9.76 & 9.76 & 9.76 \\
\hline FLC (Mbps) & 0.316 & 1.072 & 2.756 & 5.12 & 7.558 & 9.76 & 9.76 \\
\hline $\begin{array}{c}\text { ANN } \\
(\mathrm{Mbps})\end{array}$ & 0.972 & 1.919 & 4.198 & 6.848 & 8.807 & 9.76 & 9.76 \\
\hline
\end{tabular}

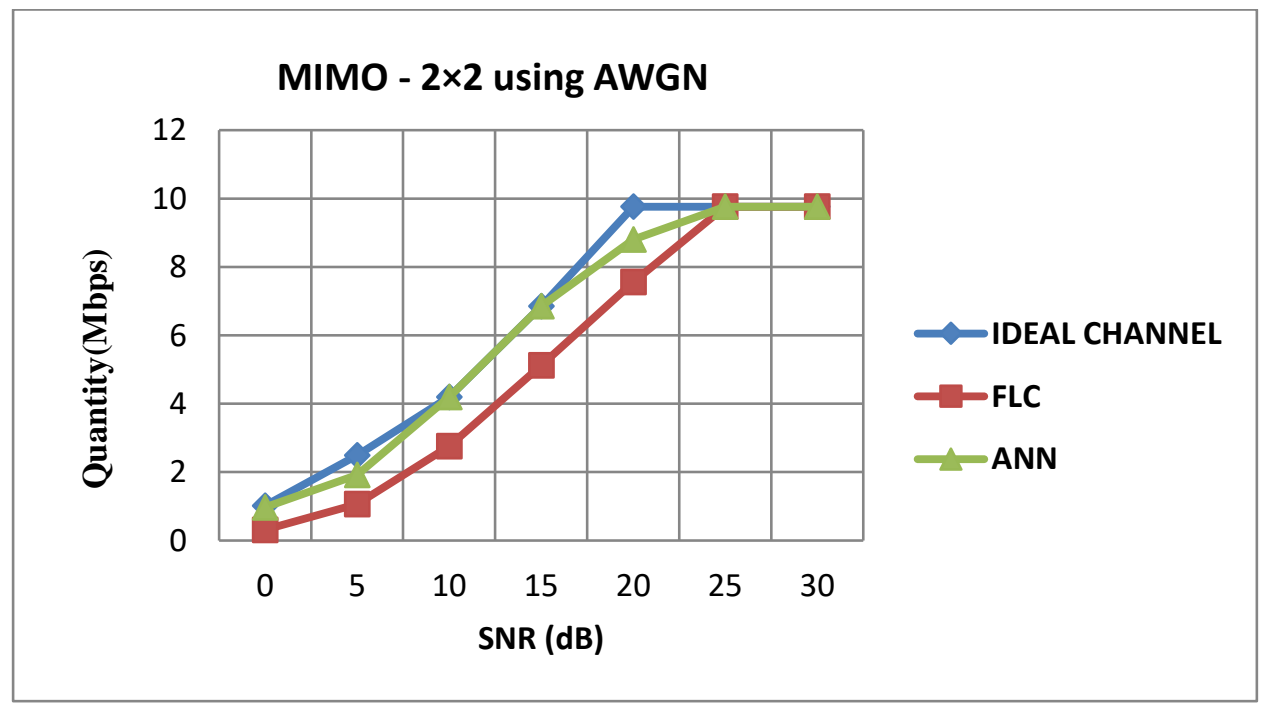

Figure 6. Estimators in contrast with CL-MIMO $-2 \times 2$ using AWGN 
Comparison between FLC algorithm and the proposed optimal channel estimation of ANN for Rayleigh and AWGN is done in this work. The channel estimation is evaluated by combinations of $2 \times 2$ and $4 \times 4$ channel noise of AWGN by CL-MIMO is given in Figure 6 and Figure 7. Table 2 and Table 3 shows the Quantity (Mbps) versus SNR(dB) results for comparing the ANN centered channel estimation method with the FLC channel estimation and the ideal channel for CL-MIMO. The FLC produces approximate efficiency of $70.42 \%$ and ANN produces average efficiency of $89.74 \%$ compared with the ideal channel over the 4x4 AWGN channel.

Table 3. MIMO - 4x4 using AWGN

\begin{tabular}{|c|c|c|c|c|c|c|c|}
\hline SNR (dB) & 0 & 5 & 10 & 15 & 20 & 25 & 30 \\
\hline $\begin{array}{c}\text { IDEAL } \\
\text { CHANNEL } \\
(\mathrm{Mbps})\end{array}$ & 2.149 & 4.886 & 7.994 & 12.99 & 14.68 & 18.51 & 18.51 \\
\hline $\begin{array}{c}\text { FLC } \\
\text { (Mbps) }\end{array}$ & 0.4112 & 2.2 & 4.964 & 9.198 & 14.08 & 18.51 & 18.51 \\
\hline $\begin{array}{c}\text { ANN } \\
(\mathrm{Mbps})\end{array}$ & 1.414 & 3.957 & 7.974 & 11.16 & 14.38 & 18.31 & 18.31 \\
\hline
\end{tabular}

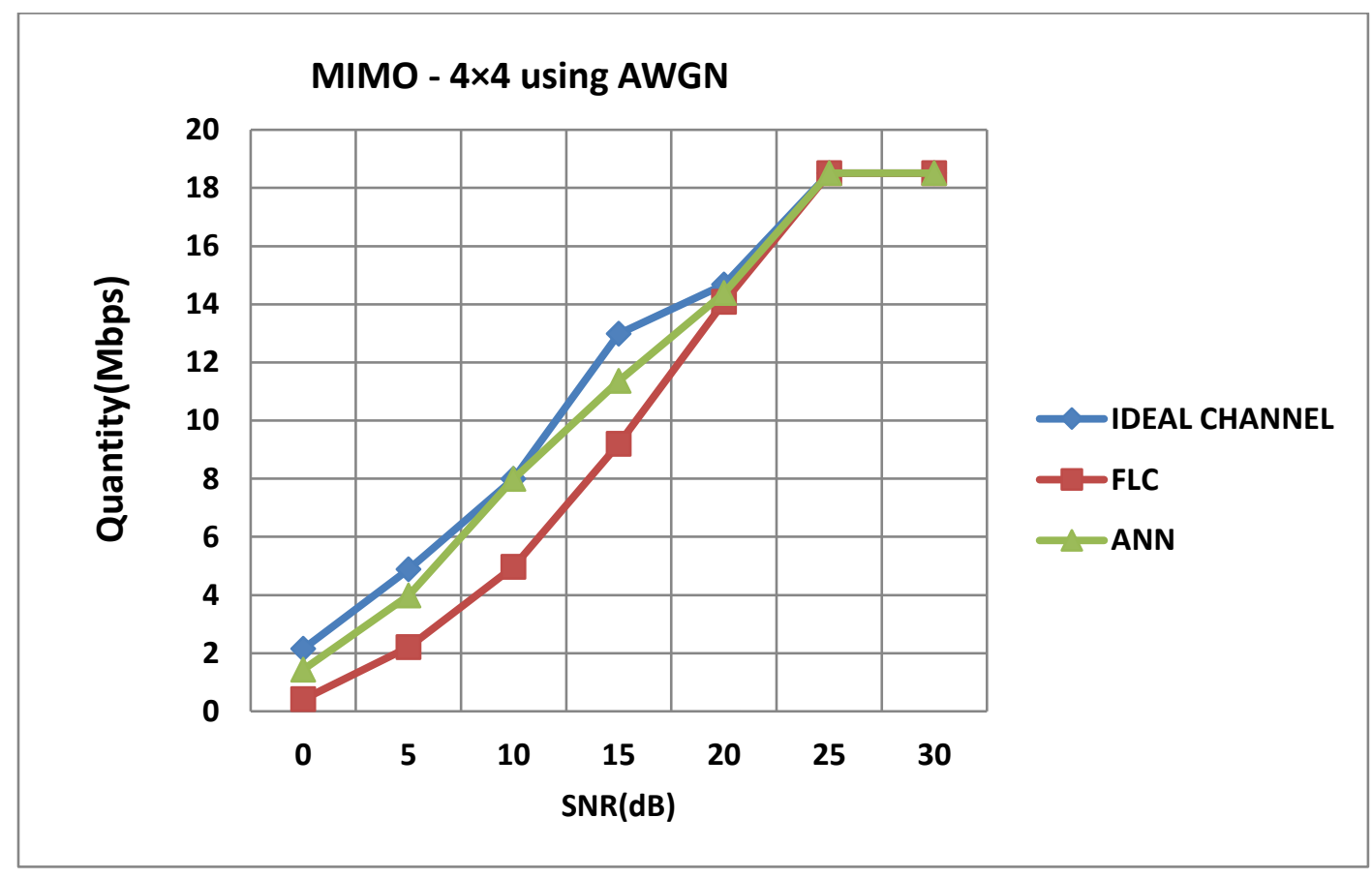

Figure 7. Estimators in contrast with MIMO - 4×4 using AWGN 
ANN framework designs and trains the most powerful input and output parameters for improving the performance and shows better results for SNR ranges from $0 \mathrm{~dB}$ to $30 \mathrm{~dB}$ compared to FLC channel estimators and ideal channel. ANN contributes nearly close to the ideal channel for CL-MIMO $(2 \times 2)$ as given in Table 2 and similarly for CL-MIMO $(4 \times 4)$ at $0,5,10,15,20,25$ and $30 \mathrm{~dB}$ respectively which gives the best result when related to FLC algorithm and ideal channel as given in Table 3. The FLC produces approximate efficiency of $76.12 \%$ and ANN produces average efficiency of $97.13 \%$ compared with the ideal channel over the $2 \times 2$ Rayleigh channel.

Table 4. CL-MIMO - 2x2 using Rayleigh

\begin{tabular}{|c|c|c|c|c|c|c|c|}
\hline SNR (dB) & 0 & 5 & 10 & 15 & 20 & 25 & 30 \\
\hline $\begin{array}{c}\text { IDEAL } \\
\text { CHANNEL } \\
(\mathrm{Mbps})\end{array}$ & 1.076 & 1.929 & 3.446 & 4.858 & 6.961 & 8.997 & 9.157 \\
\hline $\begin{array}{c}\text { FLC } \\
(\mathrm{Mbps})\end{array}$ & 0.4406 & 1.438 & 2.179 & 3.842 & 6.12 & 7.978 & 9.02 \\
\hline $\begin{array}{c}\text { ANN } \\
(\mathrm{Mbps})\end{array}$ & 1.05 & 1.873 & 3.423 & 4.59 & 6.81 & 8.456 & 9.121 \\
\hline
\end{tabular}

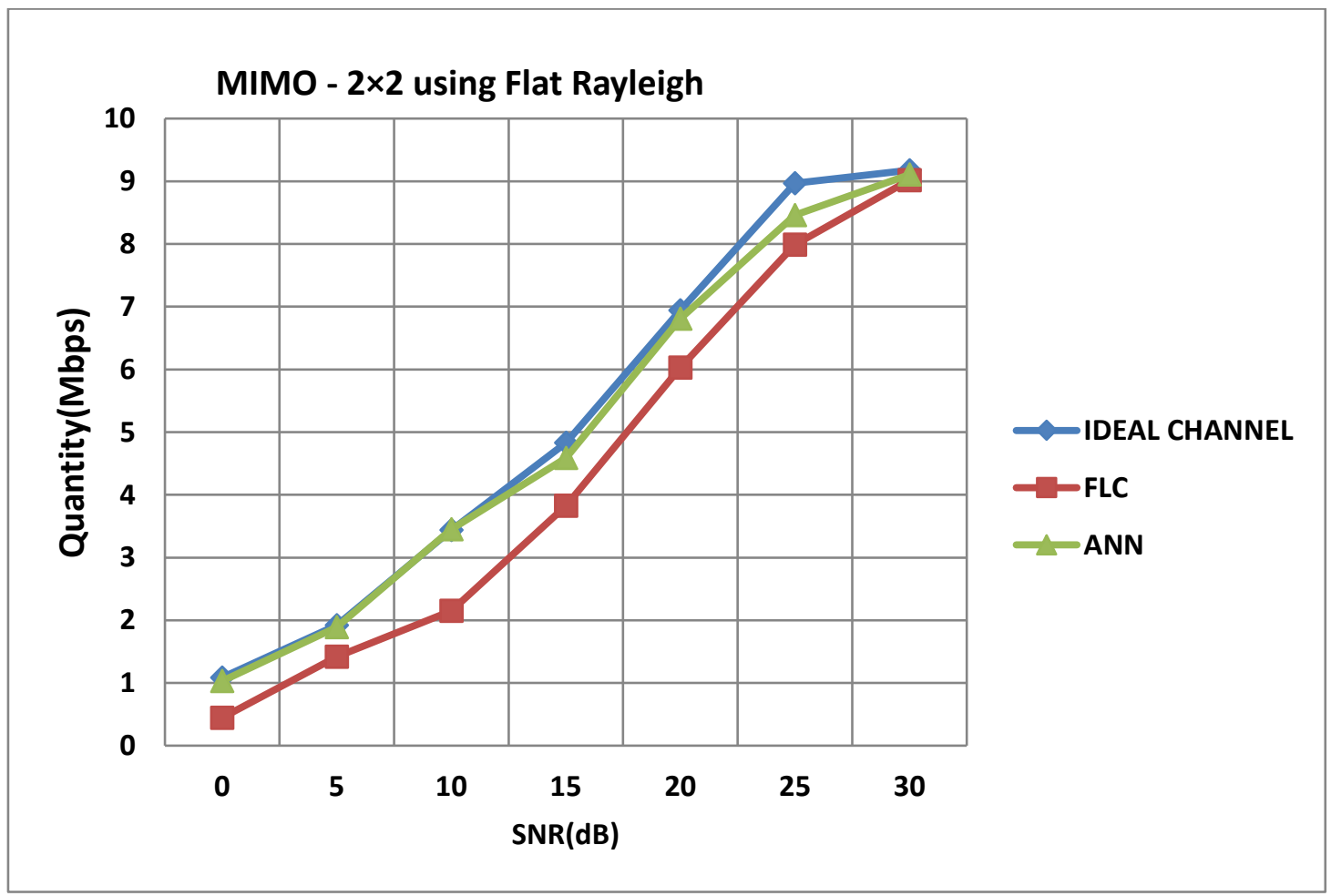


Figure 8. Estimators in contrast with CL-MIMO - $2 \times 2$ using Rayleigh

Comparison of ANN channel estimation, FLC estimation and ideal channel in CL-MIMO 2x2 and 4x4 Rayleigh Channel is shown in Figure 8 and Figure 9. In comparison to FLC based channel estimator and ideal channel, ANN explicitly propose to improve the performance and also obtain improved response for SNR range $0 \mathrm{~dB}$ to $30 \mathrm{~dB}$.

Table 5. CL-MIMO - 4x4 using Rayleigh

\begin{tabular}{|c|c|c|c|c|c|c|c|}
\hline SNR (dB) & 0 & 5 & 10 & 15 & 20 & 25 & 30 \\
\hline $\begin{array}{c}\text { IDEAL } \\
\text { CHANNEL } \\
\text { (Mbps) }\end{array}$ & 2.004 & 3.708 & 6.09 & 9.2 & 11.63 & 15.24 & 17.75 \\
\hline FLC (Mbps) & 0.9412 & 2.1 & 3.965 & 7.282 & 9.988 & 14.16 & 17.34 \\
\hline ANN (Mbps) & 1.62 & 2.94 & 5.66 & 9.012 & 11.21 & 15.08 & 17.48 \\
\hline
\end{tabular}

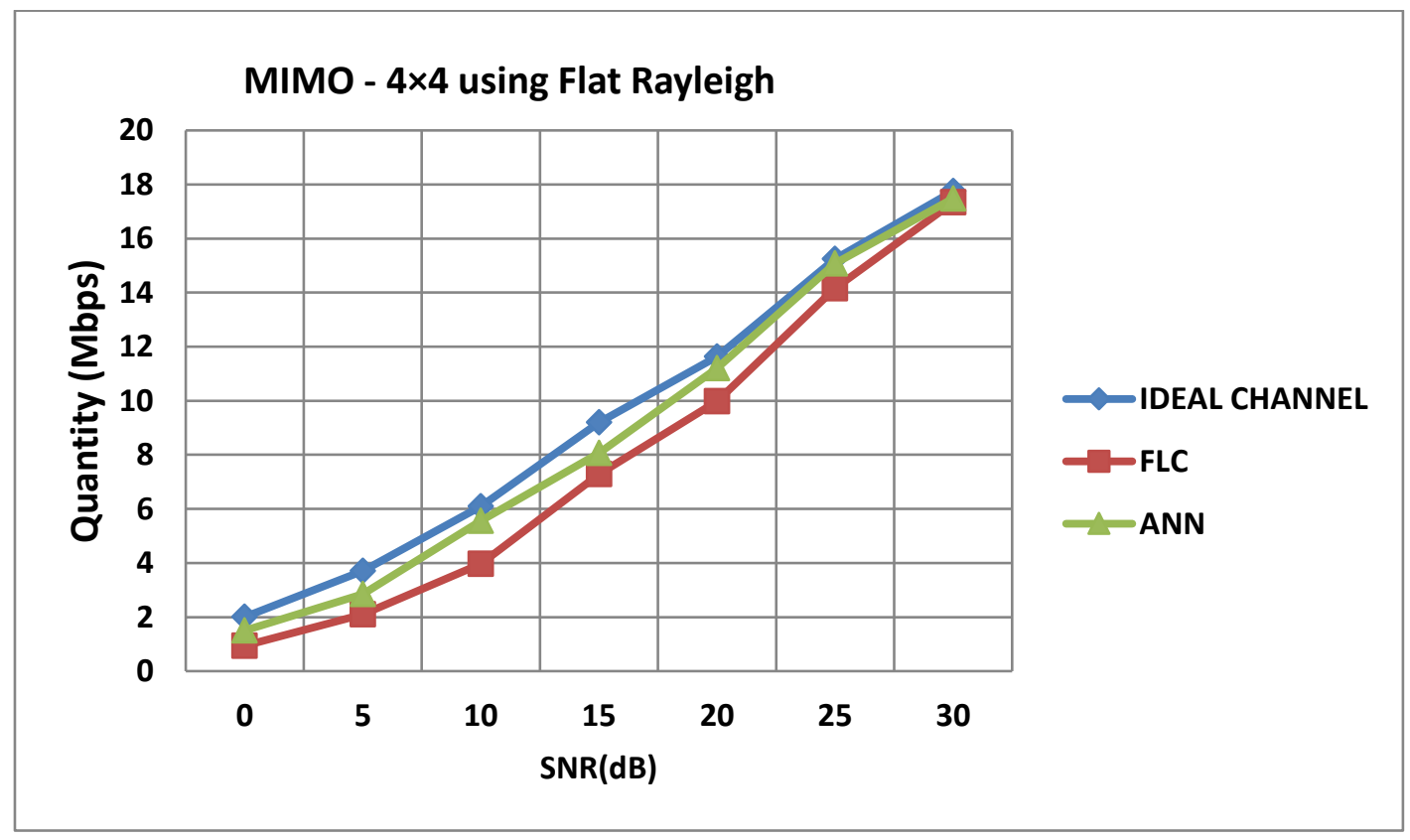

Figure 9. Comparison CL-MIMO (4×4) Rayleigh Channel 
ANN gives 1.05 Mbps, 1.873Mbps, 3.423Mbps, 4.59Mbps, 6.81Mbps, 8.456Mbps and 9.121 Mbps at 0, $5,10,15,20,25$ and $30 \mathrm{~dB}$ respectively as given in Table 4 and $1.62 \mathrm{Mbps}, 2.94 \mathrm{Mbps}, 5.66 \mathrm{Mbps}$, 9.012Mbps, 11.21Mbps, 15.28Mbps and 17.48 at Mbps at 0, 5, 10, 15, 20, 25 and $30 \mathrm{~dB}$ respectively as given in Table 5. The FLC produces approximate efficiency of $74.90 \%$ and ANN produces average efficiency of $92.11 \%$ compared with the ideal channel over the $4 \times 4$ AWGN channel. ANN channel estimation has yielded good performance compared to the FLC channel estimation and ideal channel.

\section{CONCLUSION}

ANN is used for modeling nonlinear channel estimation and relay selection scheme as an alternate to FLC based channel estimation system for AWGN and Rayleigh using 2x2 and 4x4 CL-MIMO system. SNR, $\mathrm{CG}_{\mathrm{i}}$ and $\mathrm{h}_{\mathrm{i}}$ were used as the fuzzy input parameters framing 45 set of fuzzy rules and giving the finest relay after defuzzification using centroid method in the FLC relay selection system. The same set of input parameters used in FLC was utilized to train the ANN based channel estimation system. Among 1250 patterns of parameters, nearly $70 \%$ of samples were used for training and the left behind $30 \%$ samples were used for testing and validation of ANN based channel estimation system. ANN channel estimation and FLC channel estimation for 2x2 and 4x4 CL-MIMO of AWGN channel shows that ANN is performing similar to the ideal channel when compared with FLC. Similarly for 2x2 and 4x4 CL-MIMO of Rayleigh channel shows that ANN is very much identical to ideal channel compared to FLC in SNR range of OdB to $30 \mathrm{~dB}$. Duration for training ANN can be improved by using enhanced hybrid techniques. In future, timing and carrier synchronization at the receiver side can also be incorporated in ANN based estimation system.

\section{REFERENCES}

[1] Politis, C., et al., (2004). Cooperative networks for the future wireless world. IEEE Communications Magazine. 42(9), 70-79.

[2] Laneman, J.N., D.N. Tse, and G.W. Wornell, (2004) Cooperative diversity in wireless networks: Efficient protocols and outage behaviour. IEEE Transactions on Information theory. 50(12), 3062-3080.

[3] Kharat, P. and J. Gavade, (2013), Cooperative communication: New trend in wireless communication. International Journal of Future Generation Communication and Networking., 6(5), 157-166.

[5] Laneman, J.N. and G.W. Wornell,(2003), Distributed space-time-coded protocols for exploiting cooperative diversity in wireless networks. IEEE Transactions on Information theory. 49(10), 2415-2425.

[5] Hasna, M.O. and M.S. Alouini, (2003), End-to-end performance of transmission systems with relays over Rayleigh-fading channels. IEEE Transactions on Wireless Communications. 2(6), 1126-1131. 
[6] Sowjanya Ponnaluru and Satyanaraya Penke, (2020), Deep learning for estimating the channel in orthogonal frequency division multiplexing systems. Journal of Ambient Intelligence and Humanized Computing. https://doi.org/10.1007/s12652-020-02010-1

[7] Zhao, Y., R. Adve, and T.J. Lim, (2006), Symbol error rate of selection amplify and forward relay systems. IEEE Communications Letters. 10(11), 757-759.

[8] Krikidis, I., et al., (2008), Amplify-and-forward with partial relay selection. IEEE Communications Letters. 12(4), 235-237.

[9] Ezhilazhagan C and Ramakrishnan M, (2017), Estimation of MTOs and Channel parameters for Multi-Relay Cooperative Network in Wireless Communication. Perspectivas em Ciencia da Informacao. 22(4), 181-190.

[10] Duong, T.Q., V.N.Q. Bao, and H.J. Zepernick,(2009), On the performance of selection decode-and-forward relay networks over Nakagami-m fading channels. IEEE Communications Letters. 13(3), 172-174.

[11] Su, W., A.K. Sadek, and K.R. Liu (2005), SER performance analysis and optimum power allocation for decode-and-forward cooperation protocol in wireless networks. IEEE Wireless Communications and Networking Conference.

[12] Ezhilazhagan, C., Ramakrishnan, M., et al., (2015), Estimating Timing and Frequency Offset along with Channel in Multi-Relay Cooperative Networks using Amplify and Forward Network. Middle East Journal of Scientific Research \& IDOSI Publications. 23, 135-140. [13] Sureshkumar, K., Ezhilazhagan, C and Ramakrishnan, M, (2015), Timing and Carrier Synchronization by Offset Estimation for Cooperative Relay Networks. Indian Journal of Science and Technology. 8(S9), 273-278.

[14] Wenyuan Wang, Yue Xiu and Zhongpei Zhang, (2019), FDD massive MIMO downlink channel estimation with complex hybrid generalized approximate message passing algorithm. Journal of Ambient Intelligence and Humanized Computing. 10, 1769-1786.

[15] Luo, J., et al., (2007), Decode-and-forward cooperative diversity with power allocation in wireless networks. IEEE transactions on Wireless Communications. 6(3), 793-799.

[16] Wang, C. L \& S.J. Syue. (2009), A geographic-based approach to relay selection or wireless and HOC relay networks, VTC Spring 2009,IEEE.

[17] Tanoli, U., et al., (2012), "Performance analysis of cooperative networks with intersrelay communication over Nakagami-m and rician fading channels". International Journal on Multidisciplinary sciences. 3(4), 24-29.

[18] Tanoli, U., et al., (2013), "Comparative analysis of fixed-gain relaying schemes for inter-relay communication over Nakagami-m fading channel”, Sindh University Research Journal-SURJ (Science Series). 45(1).

[19] Rong Wang, Jingye Cai, Xiang Yu and Jianchun Jiang, (2019), Temporal-Correlationbased compressive channel estimation for universal filtered multicarrier system over fast-fading channels, Journal of Ambient Intelligence and Humanized Computing. 10, 1681-1692.

[20] Madan, R., et al., (2008), Energy efficient cooperative relaying over fading channels with simple relay selection. IEEE Transactions on Wireless Communications. 7(8), 3013-3025. 
[21] Satish K. Shah, Pooja S. Suratia, Nirmalkumar S. Reshamwala, (2013), Comparative Performance Analysis of ANN Based MIMO Channel Estimation for downlink LTE- Advanced System employing Genetic Algorithm, Proceeding of International Conference On Soft Computing and Software Engineering [SCSE'13].

[22] Nirmalkumar S. Reshamwala, Pooja S. Suratia, Satish K. Shah, (2013), "Performance Analysis of ANN Based MIMO Channel Estimation for downlink LTE-Advanced System", National Level Paper Contest called Soft Computing for processing, Security Networking and Communication: (SCPSNC_2013).

[23] Desi Pramudiwati, (2011), "LTE System Performance In Relation To Wideband Channel Properties", Wireless and Mobile Communications Group, Department of Telecommunications, Faculty of Electrical Engineering, Mathematics and Computer Science, Delft University of Technology.

[24] Nirmalkumar S. Reshamwala, Pooja S. Suratia, Satish K. Shah, (2013), "Study of ANN Configuration on Performance of Smart MIMO Channel Estimation for Downlink LTEAdvanced System", International Journal of Computer Network and Information Society.5(11), 27-35.

[25] [Online].Available: http://www.nt.tuwien.ac.at/about-us/staff/josep-colom-ikuno/ltesimulators/

[26] C. Mehlfuhrer, J. Colom Ikuno, M. Simko, S. Schwarz, M. Wrulich, M. Rupp, (2011), The Vienna LTE Simulators - Enabling Reproducibility in Wireless Communications Research, EURASIP Journal on Advances in Signal Processing. 1-13.

[27] Farooq Khan, (2009), LTE for 4G Mobile Broadband Air Interface Technologies and Performance, Telecom R\&D Center Samsung Telecommunications, America, Cambridge University Press.

C.EZHILAZHAGAN received B.E. degree in Electronics and Communication Engineering and M.E. degree in Optical Communication in Anna University, Chennai. His areas of interests wireless communication, Mobile ad hoc Networks and Sensor Networks. He is currently pursuing Ph.D in the area of Wireless Communication in Anna University, Chennai. He has a total experience in teaching of more than 11 years. He has published a book titled Optical Communication and Networking and has published 3 patents. He has published 4 international journals, 4 international conferences and 3 national conferences. He is currently working as Assistant Professor in Department of Electronics and Communication Engineering, Dr.NGP

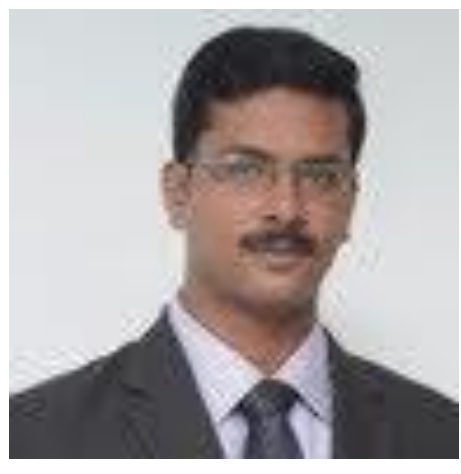
Institute of Technology, Kalapatti Road, Coimbatore-641 048. 
Dr.M.Ramakrishnan received B.E. degree in Electronics and Communication Engineering from Bharathiar University and M.E. degree in Applied Electronics from Madras University. He received his Doctorate in Faculty of Information and Communication from Anna University. He has a vast experience of 12 years in Industry and more than 25 years in teaching. His areas of interest are MANETS and Wireless Communication. He has published more than 10 International Journals, 3 National Journals and 19 Conferences and 1 patent. He is presently working as Dean (Innovation) and Professor, Sethu Institute of Technology, Kariapatti, Virudhunagar District-626115, Tamil Nadu, India.

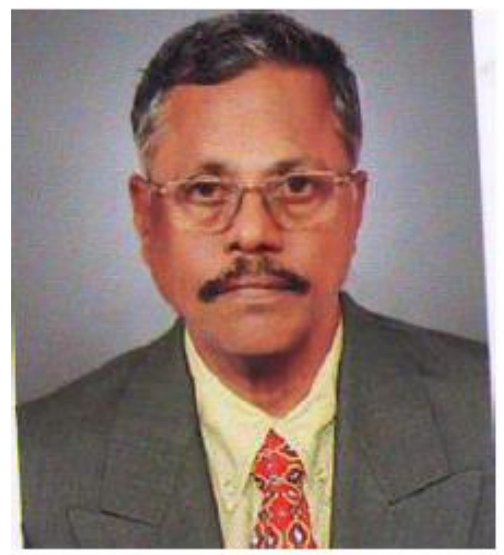

